\section{Diagnosis of an abnormality of the left main coronary}

A 74-year-old lady presented with chest pain 2 months after PCI treatment of a non-ST elevation myocardial infarction. Her past medical history was notable for systemic lupus erythematosus, hypertension and hypercholesterolaemia.

During the previous percutaneous coronary intervention (PCI), both the right coronary artery and left anterior descending (LAD) had been treated with stents.

During this procedure, the left main stem (LMS) was noted to have minor irregularity (figure $1 \mathrm{~A}, \mathrm{~B}$ ) but this was not considered to be obstructive.

She had been pain-free for 2 months, but had sudden onset of pain, and coronary angiography was undertaken. Coronary angiography demonstrated an unexpected linear lesion within the LMS (figure 1C-E) apparent in multiple views. Optimal therapy was discussed by the Heart Team MDT,

\section{QUESTION}

but what is the most likely cause of the apparent change in the LMS?

A. Artefact related to the radiographic projection and administration of intracoronary glyceryl trinitrate.

B. Iatrogenic dissection of the LMS caused by the stent procedure 2 months earlier.

C. Rupture of a non-obstructive atheromatous plaque.

D. Spontaneous dissection of the LMS.

For the answer see page 893

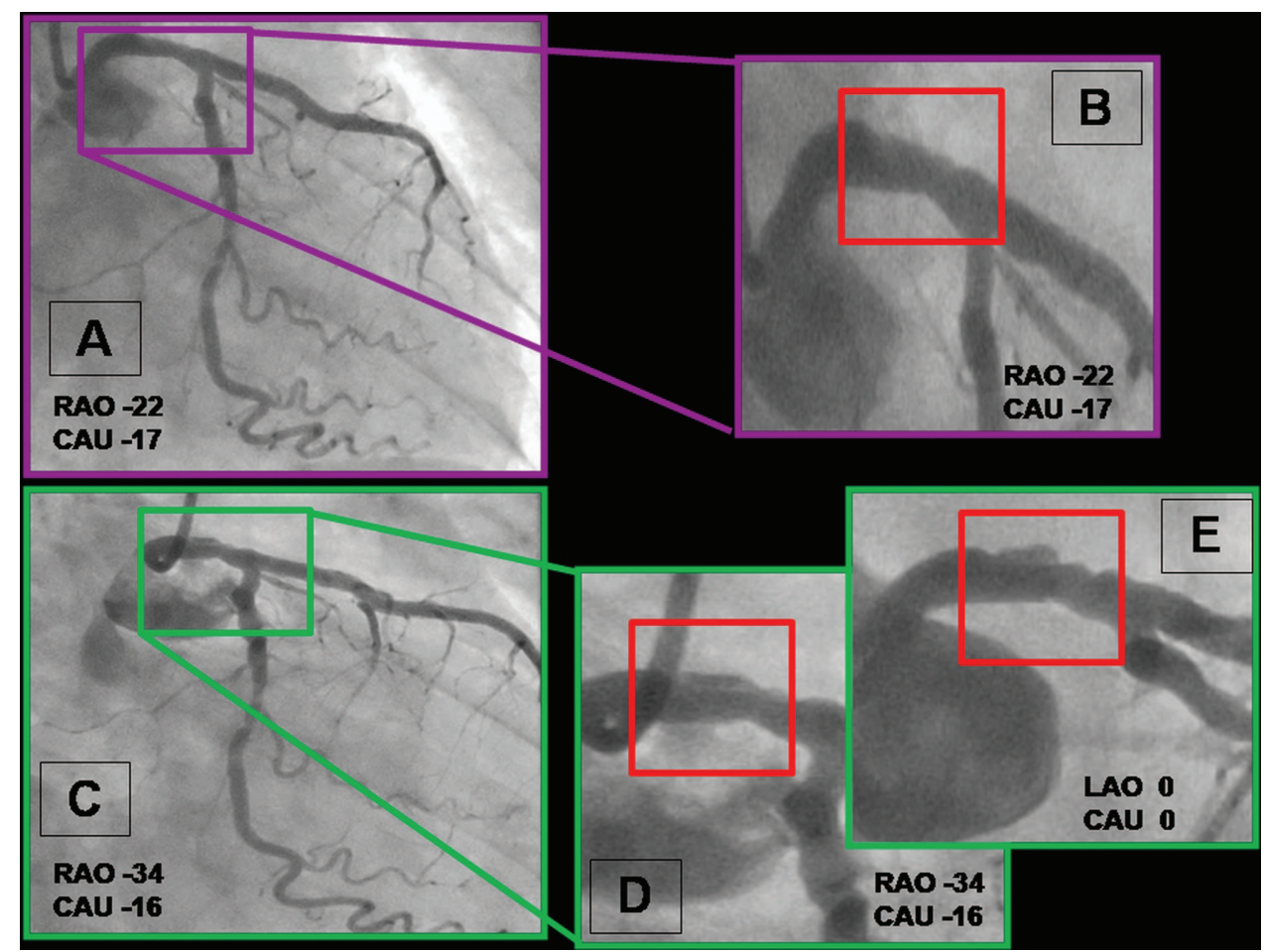

Figure 1 Coronary angiography revealing moderate disease in the left main stem ( $A$ and $B$ ) which rapidly progressed in 2 months time (C-E). 


\section{ANSWER: C}

From the question on page 891

This diagnosis can be made by careful consideration and exclusion of the other diagnostic possibilities. Careful examination of the LMS in orthogonal views noting the persistence of the abnormality excludes radiographic projection artefact. Vasodilation after glyceryl trinitrate generally produces uniform vessel dilation without luminal abnormality.

A stent-related dissection would usually be evident on the procedural films and would relate to the proximal edge of the stent in the LAD rather than being geographically distinct.

Spontaneous coronary dissection typically occurs in nonatherosclerotic coronary arteries of younger women. The angiographic appearances usually reflect mural haematoma with luminal compression. ${ }^{1}$ When linear luminal filling defects occur, they involve longer coronary segments and are usually obstructive to antegrade flow. In this case, these characteristic features are absent, and the angiogram shows a diverticulum within the wall of LMS filling with contrast. Consequently, these appearances are much more likely to be related to a ruptured plaque with underlying cholesterol-rich material being washed out by circulating blood leaving a cavity within the vessel wall.

To confirm this, the diagnosis was clarified by the aid of frequency-domain optical coherence tomography (FD-OCT (Ilumien Optis PCI Optimization System, St Jude Medical, St Paul, Minnesota, USA) (figure 2A-D) which demonstrated a large ruptured atheromatous ulcer confluent with the lumen. This appearance contrasted with that of either spontaneous or mechanical dissection in which a true lumen is surrounded and compressed by a false lumen represented by adventitia and not by the intima/media demonstrated by OCT in this case. ${ }^{2} 3$

Giovanni Luigi De Maria, ${ }^{1,2}$ Neil Ruparelia, ${ }^{1}$ Rajesh Kharbanda, ${ }^{1}$ Adrian Banning ${ }^{1}$

${ }^{1}$ Oxford University Hospitals NHS Trust, Oxford Heart Centre, Oxford, UK ${ }^{2}$ Cardiovascular Medicine Department, Catholic University of the Sacred Heart, Rome, Italy

Correspondence to Professor Adrian P Banning, Oxford University Hospitals NHS Trust, Oxford Heart Centre, Headley Way, Oxford OX39DU, UK; adrian.banning@ouh.nhs.uk

Contributors $A B$ and RK performed the procedure and images analysis. GLDM and NR drafted the article. $A B$ revised the manuscript. All the authors gave final approval.

Competing interests None.

Patient consent Obtained.

Provenance and peer review Not commissioned; internally peer reviewed.

To cite De Maria GL, Ruparelia N, Kharbanda R, et al. Heart 2014;100:893.

Received 19 December 2013

Revised 27 December 2013

Accepted 2 January 2014

Heart 2014;100:893. doi:10.1136/heartjnl-2013-305432

\section{REFERENCES}

1 Saw J. Spontaneous coronary artery dissection. Can J Cardiol 2013;29:1027-33.

2 Adlam D, Cuculi F, Banning A. Management of spontaneous coronary artery dissection in the primary percutaneous coronary intervention era. I Invasive Cardiol 2010;22:549-53.

3 Ruiz-García J, Jiménez-Valero S, Salinas P, et al. Optical coherence tomography in spontaneous coronary dissection and in the complications following percutaneous treatment. Rev Esp Cardiol 2013;66:72-3.

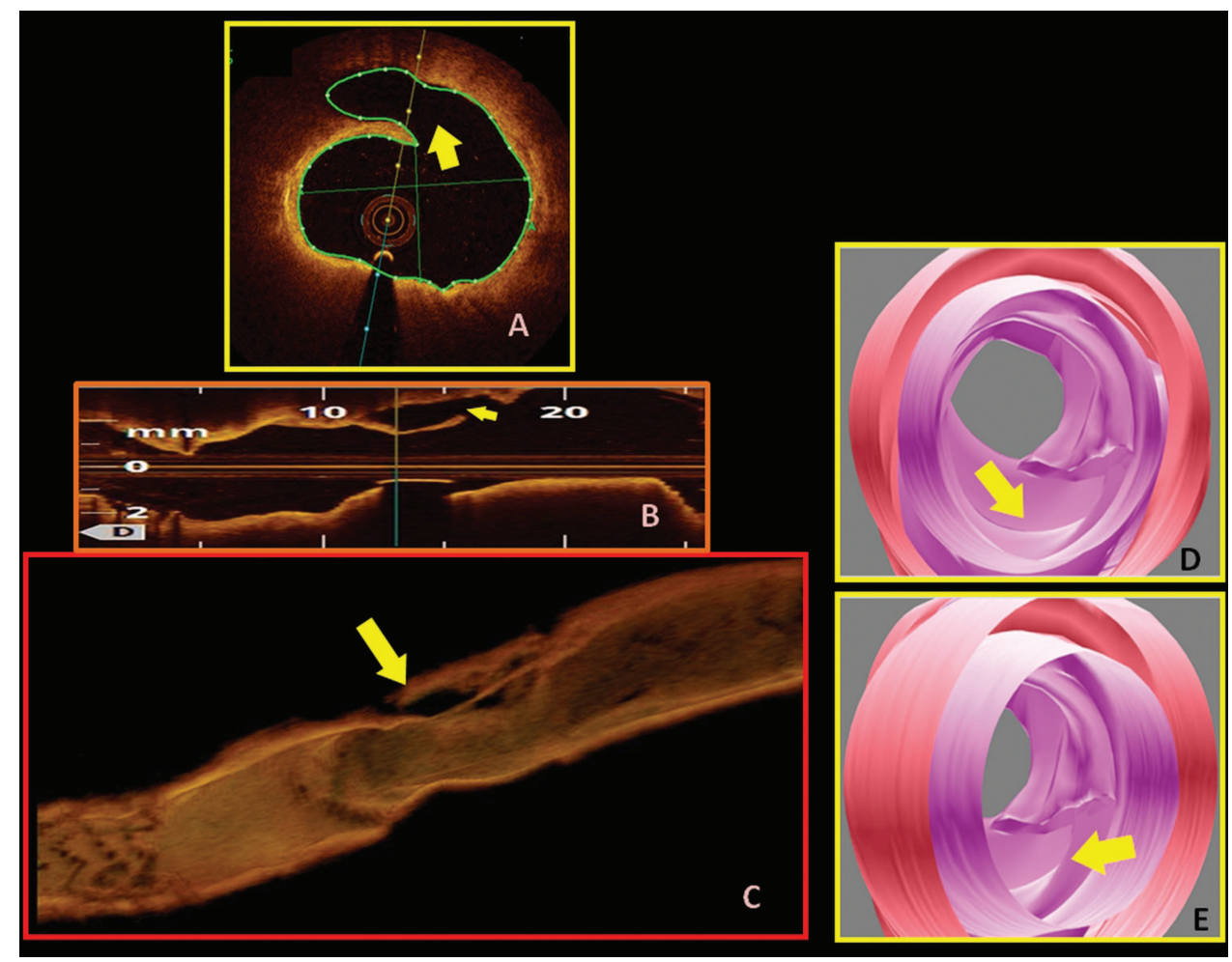

Figure 2 Evidence at FD-OCT (A-C) and on three-dimensional (3D) reconstruction (D) of an ulcerated atheroma (yellow arrow) and persistent invaginations (white arrows). 